Article

\title{
Anti-Inflammatory Effects of Phlebia sp. Extract in Lipopoly- saccharide-stimulated RAW 264.7 Macrophages
}

\author{
Eui Hyeon Lim ${ }^{1}$, Seul-Ki Mun ${ }^{1}$, Jong-Jin Kim ${ }^{2}$, Dong-Jo Chang ${ }^{1}$ and Sung-Tae Yee 1,* \\ 1 Department of Pharmacy, Sunchon National University, 255 Jungang-Ro, Suncheon 57922, Korea; \\ sksms147zld@naver.com (E.H.L.); motomoto1210@naver.com (S.-K.M.); djchang@scnu.ac.kr (D.-J.C.) \\ 2 Department of Biology, Sunchon National University, 255 Jungang-Ro, Suncheon 57922, Korea; \\ kimij@scnu.ac.kr (J.-J.K.) \\ * Correspondence: sungtae@sunchon.ac.kr; Tel.: +82-61-750-3752; Fax: +82-61-750-3708
}

\begin{abstract}
Lichens are a life form in which algae and fungi have a symbiotic relationship. A lichen has various biological activities, including anti-inflammatory and anti-proliferative activities. Inflammation is a response caused by various factors, such as infection by pathogens or tissue damage; excessive reactions can contribute to the etiology of chronic diseases, such as asthma, brain damage, and serious tissue damage. This study demonstrates the anti-inflammatory effect of ethyl acetate extract from Phlebia sp. on NF- $\mathrm{kB}$ and AP-1 pathways in the lipopolysaccharide-treated RAW 264.7 cell. Especially, Phlebia sp. extract inhibits the phosphorylation of AP-1 signaling (c-Fos and c-Jun) and its upstream MKK/MAPKs (MKK4, MKK7 and JNK), which induced a decrease in the production of the inflammatory cytokines such as tumor necrosis factor- $\alpha$ (TNF- $\alpha$ ), interleukin-6 (IL-6), interleukin-1 $\beta$ (IL-1 $\beta$ ), and granulocyte-macrophage colony-stimulating factor (GM-CSF) in downstream of AP-1 signaling. Furthermore, Phlebia sp. extract inhibited the production of final inflammatory effector molecules involved in AP-1 signaling, including nitric oxide (NO) and prostaglandin E2 (PGE2). Here, we report that Phlebia sp. extract has the potential to be developed as an antiinflammatory agent.
\end{abstract}

Keywords: Anti-inflammation; Phlebia sp; RAW264.7 macrophages; AP-1; cytokines

\section{Introduction}

Macrophages play a critical role in the immune system by serving as the first line of defense against pathogens and tumors and also link innate and adaptive immunity [1]. Macrophages, one of antigen-presenting cells (APCs), prime the differentiation of naive CD4 and CD8 T cells into subtype effector T cells by secreting various cytokines, and act as a critical costimulatory molecule for the efficient T cells activation [2].

Inflammation can be initiated and maintained by macrophages. In inflammation, macrophages secrete nitric oxide (NO) and various inflammatory mediators such as cytokines, and prostaglandin E2 (PGE2), which finally leads the infiltration of immune cells, tissues damage, pain, and swelling. Macrophages are differentiated from monocytes in the blood and then move into peripheral tissues to surveil the invasion of pathogens [3]. For this reason, suppression of NF- $\mathrm{kB}$ and/or AP-1 signaling has been regarded as one of best strategies for treatment of inflammation.

Lichens are complex organisms composed of fungi and photosynthetic partners (algae or cyanobacteria) [4]. Lichens are characterized by their sensitivity to environmental stresses, such as pollution and climatic changes [5]. Moreover, lichens have been used as food ingredients and herbal tea in Japan and China [6]. Lichens produce secondary metabolites that have a variety of biological activities, such as anti-inflammatory, antitumor, anti-oxidant, and immunosuppressive activities [6-8]. Phlebia sp. is an endolichenic 
fungi constituting the lichen, Peltigera neopolydactyla. and belongs to the family Meruliaceae and the order Polyporales [9].

There have been no reports of the anti-inflammatory effects of Phlebia sp. extracts (PSE). Herein, we report that PSE inhibits AP-1 signaling on the activated-macrophage by regulating mitogen-activated protein kinase kinases (MKK) and mitogen-activated protein kinases (MAPKs). Effector molecules in downstream of AP-1 signaling, including inflammatory cytokines (TNF-a, IL-1b, Il-6, GM-CSF), NO, and PGE2, are decreased by treatment of PSE on LPS-stimulated RAW 264.7 cells. Based on our results, we conclude that PSE can be used for the development of material and functional products for antiinflammation.

\section{Materials and Methods}

\subsection{Sample Collection, Identification and Extraction}

Peltigera neopolydactyla was collected from the coastal rocks of southern Korea in 2018 and deposited at the Korean Lichen and Allied Bioresource Center (KOLABIC) of the Korean Lichen Research Institute (KoLRI), Sunchon National University, Korea. For the accurate classification of the fungal part of the sample, the ribosomal DNA internal transcribed spacer region (ITS) was analyzed to confirm that it was Phlebia sp. To obtain the ethyl acetate (EA) PSE, Phlebia sp. was cultured on malt extract agar. Then, three to four fungal agar pieces were obtained and placed in $200 \mathrm{~mL}$ of Malt and Yeast Extract Broth (MY) and incubated for one month with shaking at a $150 \mathrm{rpm}$ at $15^{\circ} \mathrm{C}$. After adding an equivalent amount of EA to the culture for $2 \mathrm{~h}$, the culture was thoroughly mixed, filtered using $3 \mathrm{M}$ filter paper, and the water/EA layer was separated. The EA layer obtained through this process was evaporated using a vacuum rotary evaporator, and $5 \mathrm{~mL}$ of EA was added to the evaporated product. The product was subsequently sonicated, and the EA was evaporated in an oven. The product was then stored as powder in samples vials.

\subsection{Chemicals and reagents}

Fetal bovine serum (FBS) and Roswell Park Memorial Institute 1640 medium (RPMI 1640) were purchased from Hyclone Laboratories (Hyclone, South Logan, UT, USA). Dimethyl sulfoxide (DMSO), 2-mercaptoehanol and lipopolysaccharides (Escherichia coli 0111:B4) were purchased from Sigma-Aldrich (St. Louis, MO, USA). The Cell counting Kit8 (CCK-8) was purchased from Dojindo Laboratories (Dojindo, Kumamoto, Japan). Purified hamster anti-mouse (TNF- $\alpha$ ), purified rat anti-mouse (IL-6, GM-CSF), biotin human anti-mouse (TNF- $\alpha$ ), and biotin rat anti-mouse (IL-6, GM-CSF) were purchased from BD Biosciences (San Jose, CA, USA). The IL-1 $\beta$ ELISA kit was acquired from Thermo Fisher (Rockford, IL, USA). The PGE2 ELISA kit was purchased from R\&D Systems (Minneapolis, MN, USA).

\subsection{Cell culture}

RAW 264.7 (murine macrophage cell line, KCLB 40071) cell line was acquired from the Korean Cell Line Bank (Seoul, South Korea). The cells were cultured in RPMI 1640 supplemented with $10 \%$ fetal bovine serum, 100 units $/ \mathrm{mL}$ penicillin, $100 \mu \mathrm{g} / \mathrm{mL}$ streptomycin (Invitrogen, Carlsbad, CA, USA), and 2-mercaptoethanol $(50 \mu \mathrm{M})$, in a humidified atmosphere at $37^{\circ} \mathrm{C}$ with $5 \% \mathrm{CO}_{2}$.

\subsection{Cytotoxicity assay and NO Production}

RAW 264.7 cells $\left(5 \times 10^{4}\right.$ cells/well) were seeded in 96-well plates and incubated overnight. The next day, each well was pre-incubated with LPS $(1 \mu \mathrm{g} / \mathrm{mL})$ for $1 \mathrm{~h}$ and treated with $1,3,10,30$ and $50 \mu \mathrm{g} / \mathrm{mL}$ of the endolichenic fungi for $24 \mathrm{~h}$ at $5 \% \mathrm{CO}_{2}$ and $37{ }^{\circ} \mathrm{C}$. Then, the half of supernatant was removed from each well, and CCK-8 $(10 \mu \mathrm{L})$ was added to the 
wells. The optical density was measured at $450 \mathrm{~nm}$ using a microplate reader (Versa Max, Molecular Devices, Sunnyvale, CA). The supernatant was used to measure the NO production, using the Griess assay [6].

\subsection{Cytokine assay}

RAW 264.7 cells $\left(5 \times 10^{5}\right.$ cells/well) were seeded in 12 -well plates and incubated overnight. The next day, each well was pre-incubated with LPS $(1 \mu \mathrm{g} / \mathrm{mL})$ for $1 \mathrm{~h}$ and treated with $1,3,10,30$ and $50 \mu \mathrm{g} / \mathrm{mL}$ PSE, for $24 \mathrm{~h}$ at $5 \% \mathrm{CO}_{2}$ and $37^{\circ} \mathrm{C}$. Cytokine concentrations were determined by using ELISA.

\subsection{Western blot assay}

RAW 264.7 cells were incubated overnight in a 6-well plate at a density of $1 \times 10^{6}$ cells/well. Then, PSE was added to the cells and incubated for $4 \mathrm{~h}$ or $18 \mathrm{~h}$. The cells were washed once with cold phosphate-buffered saline (PBS) on ice and lysed in radioimmunoprecipitation assay (RIPA) buffer containing a phosphatase and protease inhibitor cocktail (Thermo, Rockford, IL, USA). Proteins were obtained by centrifugation at 14,000 $\times \mathrm{g}$ and 4 ${ }^{\circ} \mathrm{C}$ for $20 \mathrm{~min}$. The concentration of the protein samples was determined using a bicinchoninic acid (BCA) protein assay kit (Thermo, Rockford, IL, USA). Protein samples were separated using $4-12 \%$ bis-tris plus gels (Thermo, Rockford, IL, USA) and transferred to nitrocellulose membranes (Thermo, Rockford, IL, USA). The membranes were incubated with blocking solution for $3 \mathrm{~h}$ and then incubated overnight at $4{ }^{\circ} \mathrm{C}$ with primary antibodies. The primary antibodies included $\beta$-actin (1:2000, Thermo, Rockford, IL, USA), inducible NO synthase (iNOS), Cyclooxygenase-2 (COX-2), c-Jun, p-c-Jun, c-Fos, p-c-Fos, p38, pp38, ERK, p-ERK, JNK, p-JNK, MKK4, p-MKK4, MKK7, p-MKK7, IкB, p-IкB, IKK, p-IKK, $\mathrm{NF}-\kappa \mathrm{B}$ (p65), and p-NF- $\kappa \mathrm{B}$ (p-p65). All primary antibodies for western blot were purchased from cell signaling technology except $\beta$-actin. The membranes were washed with TBST (Thermo, Rockford, IL, USA) and incubated with horseradish peroxidase (HRP)-conjugated secondary antibody (Thermo, Rockford, IL, USA) for $3 \mathrm{~h}$ at room temperature with shaking. Membranes were treated with an enhanced chemiluminescence kit (Thermo, Rockford, IL, USA). Protein bands were captured using the ChemiDoc Imaging system (BioRad, Hercules, CA, USA).

\subsection{Statistical analysis}

Data are presented as mean \pm standard deviation (SD). Statistical differences between groups were analyzed using SPSS version 26 (SPSS, Chicago, IL, USA) followed by Student's $t$-test. $p$-values of 0.05 or less indicated significant difference.

\section{Results}

\subsection{Viability and NO inhibition effect of PSE in RAW264.7 macrophages.}

Macrophages play an important role in inflammation by injury or infections [10]. Nitric oxide (NO) has many biological functions, including the elimination of bacteria and mediation of neurotransmission. When an inflammatory occurs, NO plays dual role in immunity and inflammatory responses [11]. First, we have checked the cytotoxicity of PSE and the effect on NO production. Specifically, the anti-inflammatory activity of PSE was investigated in LPS-stimulated RAW 264.7 macrophages. The RAW 264.7 cells were exposed to varying concentrations of PSE and the cytotoxicity was measured using CCK-8 $24 \mathrm{~h}$ later (Figure 1A). As shown in Figure 1A, PSE has no cytotoxicity within treated concentrations to RAW 264.7 macrophages. In addition, the non-cytotoxic PSE has an inhibitory effect on the NO production from LPS-stimulated RAW 264.7 macrophages in concentration dependent manner (Figure 1B). 
A

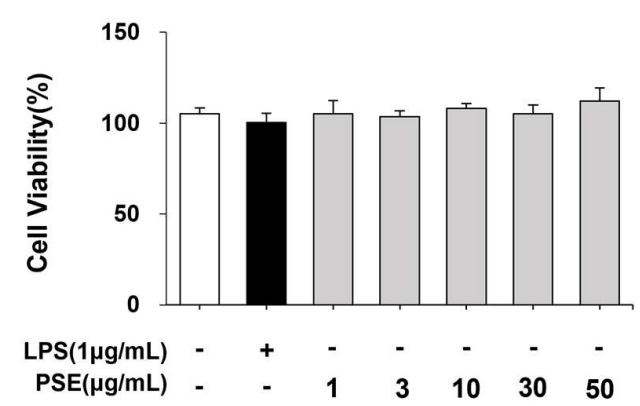

B

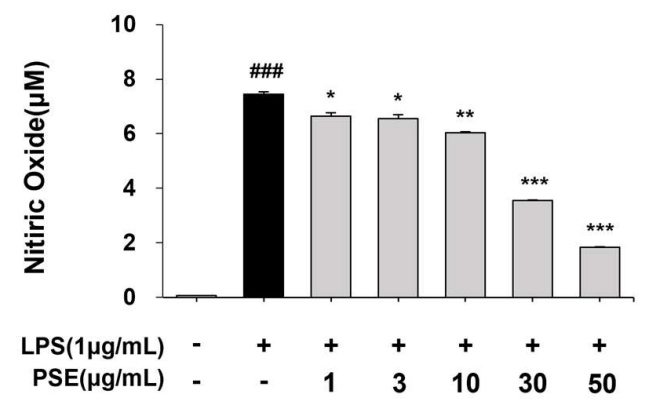

Figure 1. Confirmation of viability and NO inhibition effect of PSE in RAW264.7 macrophages. Effect of PSE on the viability of RAW 264.7 cells. RAW264.7 macrophages were treated with various concentrations of PSE to check viability. (A). The cells were pretreated with $1 \mu \mathrm{g} / \mathrm{mL}$ LPS for $1 \mathrm{~h}$ and subsequently treated with 1-50 $\mu \mathrm{g} / \mathrm{mL}$ PSE for $24 \mathrm{~h}$. Effect of PSE on the levels of nitric oxide (NO) produced by LPS-stimulated RAW 264.7 cells. The culture supernatant was measured by Griess assay (B). Data was analyzed using spss statistics followed by Student's $t$-test (\#\# $p<0.001$, versus the control group; ${ }^{*} p<0.05,{ }^{* *} p<0.01,{ }^{* * *} p<0.001$ versus the LPS group).

\subsection{Inhibitory effect of PES on the production of inflammatory cytokines and PGE2}

Inflammatory cytokines, such as TNF- $\alpha$, IL- 6 , and IL-1 $\beta$, play an essential role in regulating inflammation [12]. GM-CSF regulates the phagocytosis of microbial pathogens by the activation of macrophages. Furthermore, GM-CSF induces differentiation and recruitment of inflammatory cells from the bone marrow into peripheral tissue. The aforementioned cytokines elicit an immune response, and simultaneously, uncontrolled immune response exacerbates inflammation, implying regulation of inflammatory cytokines is essentially required for the control of inflammation [13]. To investigate the levels of pro-inflammatory cytokines (TNF- $\alpha$, IL-6, and IL-1 $\beta$ ), macrophages were stimulated with LPS and treated with 1,3,10,30, and $50 \mu \mathrm{g} / \mathrm{ml}$ of PSE. As presented in Figure 2A-C, the PSE treatment inhibited cytokine productions in the LPS-stimulated RAW 264.7 macrophages. Figure 2D shows a significant decrease in GM-CSF production by treating PSE. Furthermore, the PSE treatment inhibited the production of PGE2 in a concentration-dependent manner as shown in Figure 2E. The biosynthesis of PGE2, which is regulated by COX-2, is increased significantly in inflammatory tissues, where it contributes to the occurrence of acute inflammation [11]. These results clearly indicate that PSE exerts anti-inflammatory effects by regulating inflammatory cytokines and PGE2. 
A

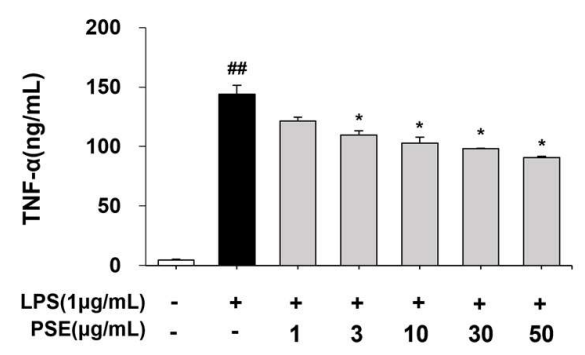

C

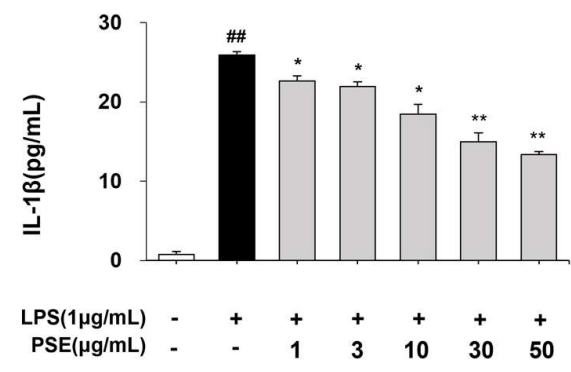

E

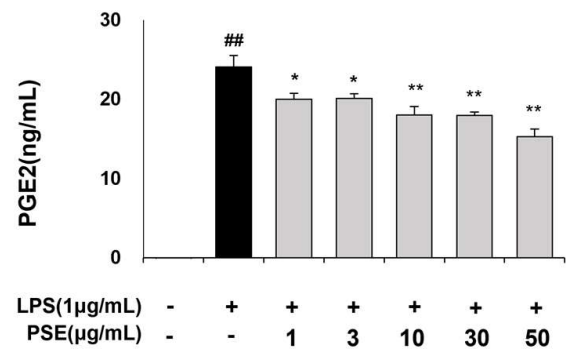

B

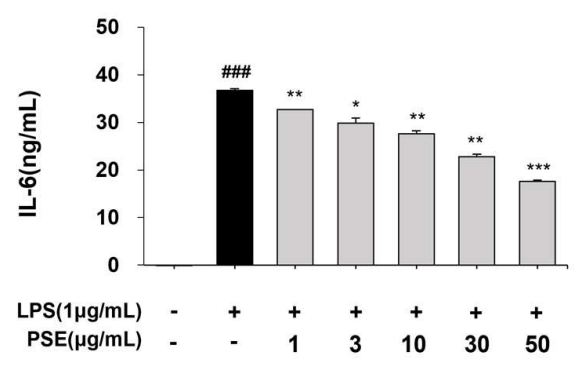

D

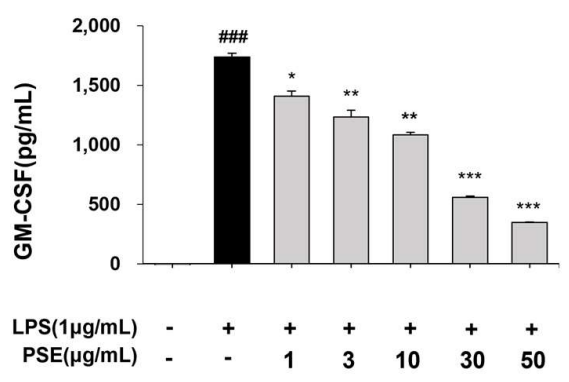

Figure 2. Inhibitory effect of PSE extract on the production of cytokines and PGE2. Effect of PSE on the pro-inflammatory cytokines and prostaglandin E2 (PGE2) produced by LPS-stimulated RAW 264.7 cells. The cells were pretreated 1 $\mu \mathrm{g} / \mathrm{mL}$ LPS for $1 \mathrm{~h}$ and treated with 1-50 $\mu \mathrm{g} / \mathrm{mL}$ PSE for $24 \mathrm{~h}$. The culture supernatant was measured by ELISA (A-E). Data was analyzed using spss statistics followed by Student's $t$-test (\# $\mathrm{p}<0.01$, \#\# $\mathrm{p}<0.001$ versus the control group; ${ }^{*} \mathrm{p}<0.05,{ }^{* *} \mathrm{p}<0.01,{ }^{* * *} \mathrm{p}<0.001$ versus the LPS group).

\subsection{Inhibitory effect of PSE on the expression of $i N O S$ and COX-2}

In the inflammatory reaction, NO secretion is regulated by iNOS in macrophages, and the generated NO molecules work as an inflammatory mediator in immunity [11]. COX-2 is another enzyme that plays a pivotal role in the mediation of inflammation and catalyzes the rate-limiting step in prostaglandin (PG) biosynthesis [14]. To check the anti-inflammatory mechanism of PSE, we conducted western blotting to confirm the expression of iNOS and COX-2. We observed a significant increase in the expression of iNOS and COX2 by treating LPS on the RAW 264.7 macrophage, whereas the treatment of PSE suppresses the expression of iNOS and COX-2 concentration-dependently (Figure 3A-C). 
A

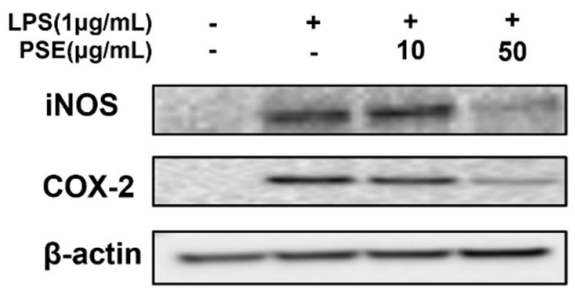

B

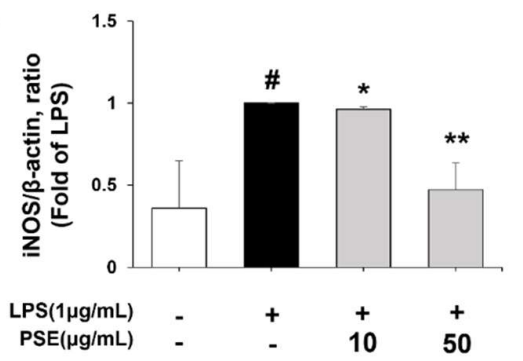

C

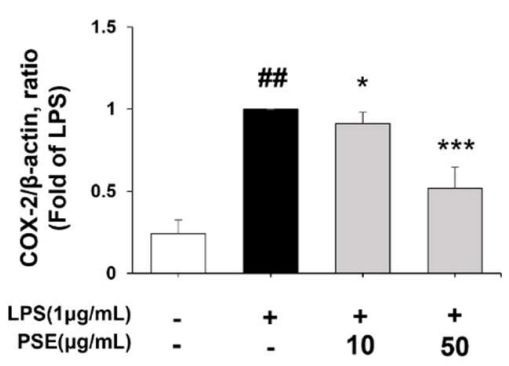

Figure 3. Inhibitory effect of PSE on the expression of iNOS and COX-2. The cells were pretreated $1 \mu \mathrm{g} / \mathrm{mL}$ LPS for $1 \mathrm{~h}$ and treated with 1-50 $\mu \mathrm{g} / \mathrm{mL}$ PSE for $17 \mathrm{~h}$. western blot analysis was performed to confirm the effect of PSE on the expression of pro-inflammatory proteins (A). Ratios of iNOS (B) and COX-2 (C) are presented as the mean \pm SD from

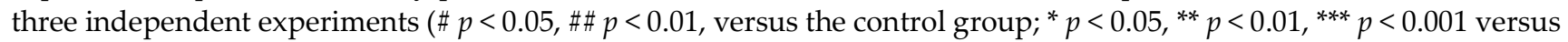
the LPS group). conclusions that can be drawn.

\subsection{Effect of the PSE on the NF- $\kappa B$ signaling pathway.}

Molecular mechanism of inflammation is very complex, and various molecules in signal transduction pathways with complicated network affect inflammation. NF- $\kappa B$ and AP-1 are well known transcription factors involved in inflammation, which regulate the gene expression of inflammatory mediators including proinflammatory cytokines and also affect the expression of genes in other key pathways related with inflammation [15]. NF$\kappa \mathrm{B}$ is bound to I $\kappa \mathrm{B}$, an inhibitor of NF- $\kappa \mathrm{B}$, and exists in an inactive state [6]. When activated by external stimulation, the inflammatory signal activates a series of I $\mathrm{KB}$ kinases known as the IкB kinase (IKK) complex [16]. After treating PSE we observed that PSE has no effect on the NF- $\kappa$ B pathway (Figure 4). Next, to find the anti-inflammatory effect of PSE, we have investigated whether PSE influenced the downstream AP-1 pathway, a TLRmediated signaling pathway. 


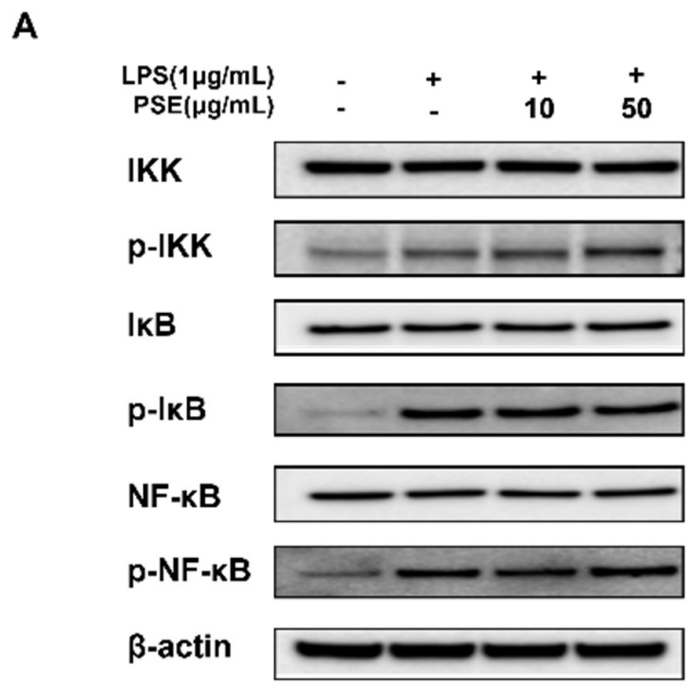

B

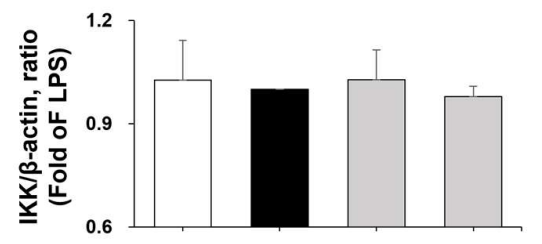

D
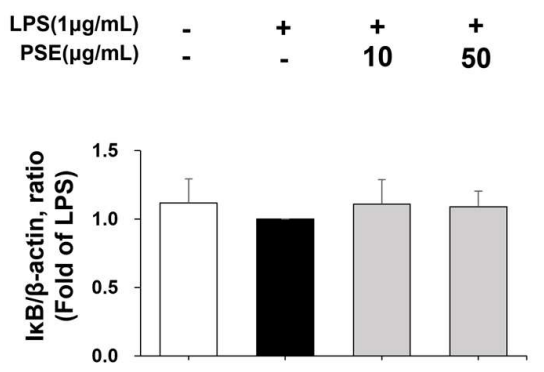

$\begin{array}{ccccc}\operatorname{LPS}(1 \mu \mathrm{g} / \mathrm{mL}) & - & + & + & + \\ \operatorname{PSE}(\mu \mathrm{g} / \mathrm{mL}) & - & - & 10 & 50\end{array}$

F

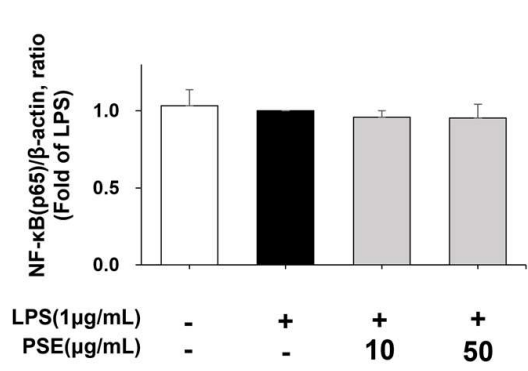

C
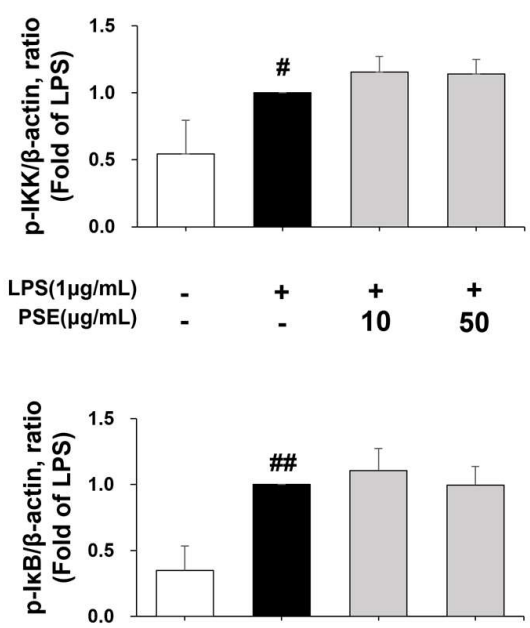

$\begin{array}{ccccc}\operatorname{LPS}(1 \mu \mathrm{g} / \mathrm{mL}) & - & + & + & + \\ \operatorname{PSE}(\mu \mathrm{g} / \mathrm{mL}) & - & - & 10 & 50\end{array}$

G

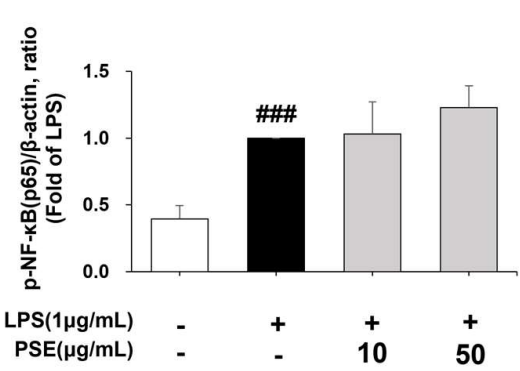

Figure 4. Effect of PSE on the activation of NF- $\kappa \mathrm{B}$ signaling. The cells were pretreated $1 \mu \mathrm{g} / \mathrm{mL}$ LPS for $1 \mathrm{~h}$ and treated with 1-50 $\mu \mathrm{g} / \mathrm{mL}$ PSE for $3 \mathrm{~h}$. Expression of IKK, p-IKK, I $\kappa \mathrm{B}, \mathrm{p}-\mathrm{I} \kappa \mathrm{B}, \mathrm{NF}-\kappa \mathrm{B}$, and p-NF- $\kappa \mathrm{B}$ were determined by western blot analysis (A). The ratios of IKK (B), p-IKK (C), I $\kappa B(D)$, p-I $\kappa B(E)$, NF- $\kappa B(F)$, and p-NF- $\kappa B(G)$ are shown. Data are presented as mean \pm SD from three independent experiments $(\# p<0.05$, \#\# $p<0.01$, \#\#\# $p<0.001$ versus the control group). 


\subsection{Anti-inflammatory activity of the PSE through inhibition of AP-1 pathway}

AP-1 is a pivotal factor in the regulation of inflammation by producing pro-inflammatory mediators and cytokines such as iNOS, COX-2, IL-1 $\beta$, IL-2, and IL-6 [17]. Phosphorylation of c-Jun, which is a member of the AP- 1 family, induces translation of gene and produces protein for TNF- $\alpha$. [18]. c-Fos is one of the most powerful transcription factors in the immune system and belongs to the Fos family. c-Fos binds to c-Jun to form active AP-1, and plays a regulatory role in inflammation [19]. LPS-induced RAW 264.7 macrophages were treated with 1,10 , and $50 \mu \mathrm{g} / \mathrm{mL}$ of PSE to confirm the effect on c-Jun and c-Fos, and their expressions were measured by western blot assay (Figure 5A). Figures 5B-E shows the quantitative analysis data with Figure $5 \mathrm{~A}$ for c-Jun and c-Fos after-treatment of the RAW264.7 macrophages with PSE. These results clearly illustrate that treatment of the macrophages with PSE suppressed the production of inflammatory proteins, which is phosphorylation of c-Jun, c-Fos and total c-Fos.

A

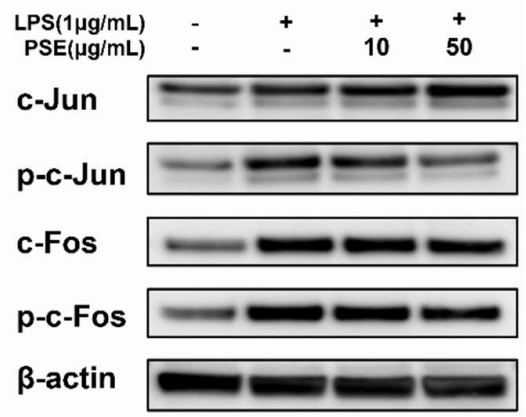

B

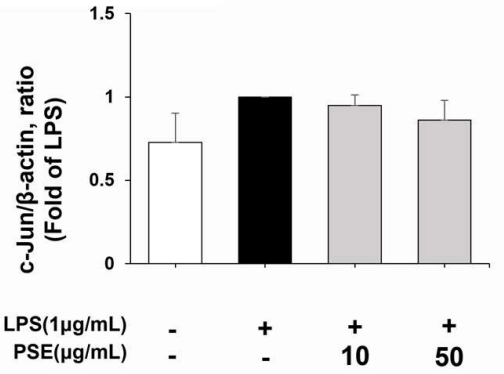

D

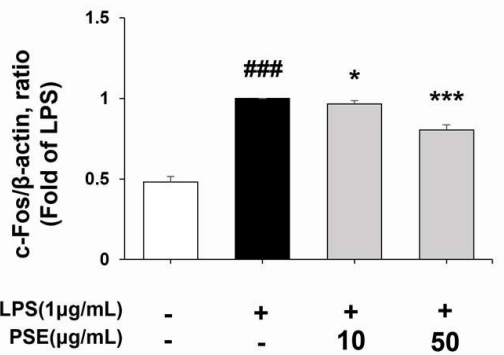

C

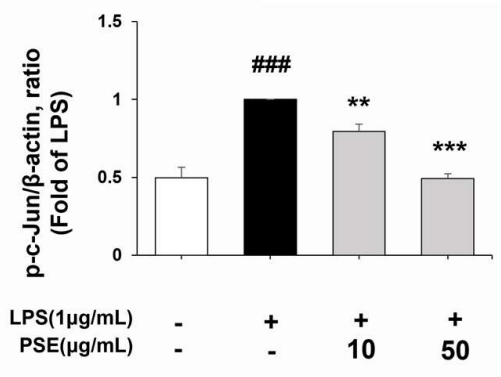

E

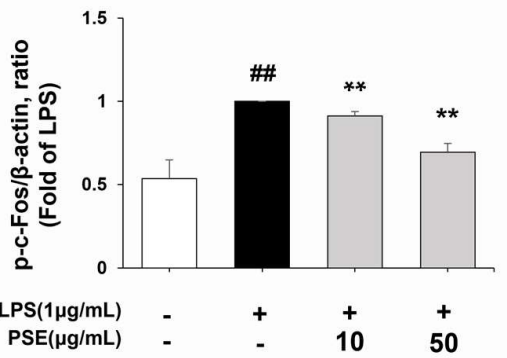

Figure 5. Inhibitory effect of PSE on the activation of AP- 1 signaling. The cells were pretreated $1 \mu \mathrm{g} / \mathrm{mL}$ LPS for $1 \mathrm{~h}$ and treated with 1-50 $\mathrm{gg} / \mathrm{mL}$ PSE for $3 \mathrm{~h}$. Expression of c-Jun, p-c-Jun, c-Fos, p-c-Fos were determined by western blot analysis (A). The ratios of c-Jun (B), p-c-Jun (C), c-Fos (D), p-c-Fos (E) are shown. Data are presented as mean \pm SD from three independent experiments (\# $p<0.05$, \#\# $p<0.01$, \#\# $p<0.001$ versus the control group; ${ }^{*} p<0.05,{ }^{* *} p<0.01,{ }^{* * *} p$ $<0.001$ versus the LPS group). 


\subsection{Anti-inflammatory activity of the PSE on the MKK/MAPKs pathway}

Activated MAPKs phosphorylate several transcription factors, such as c-Jun and c-Fos [20], and c-Jun kinase (JNK), a member of MAPKs, is a key player in inflammation as stressactivated protein kinase [21]. Numerous reports, including increase of inflammatory cytokines production by MAPKs (ERK, p38, and JNK), have demonstrated that MAPKs are important targets for inflammation regulation [21-22]. We have first investigated the inhibitory effect of the PSE on the phosphorylation of p38 and ERK, but it did not affect it (Figure 6B-E). However, phosphorylation of JNK was induced by LPS was increased, but PSE inhibits the phosphorylation of JNK concentration-dependently (Figure 6G). JNKs are activated through the sequential activation of protein kinases containing two dualspecificity MAP kinase kinases (MKK4 and MKK7) [23, 24]. We measured the effect of PSE on the phosphorylation of MKK4 and MKK7 (Figure 6I and K), showing the phosphorylation of MKK4 and MKK7 was reduced by PSE treatment. Taken together, PSE inhibits phosphorylation of MKK4 and MKK7, which result in blocking the production of inflammatory cytokines and effector molecules via decrease of phosphorylation of JNK.

A

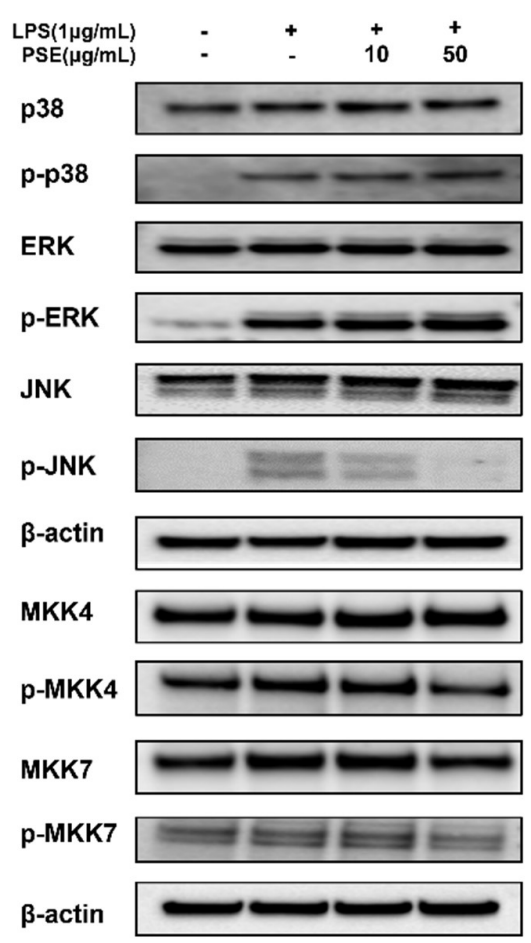

B
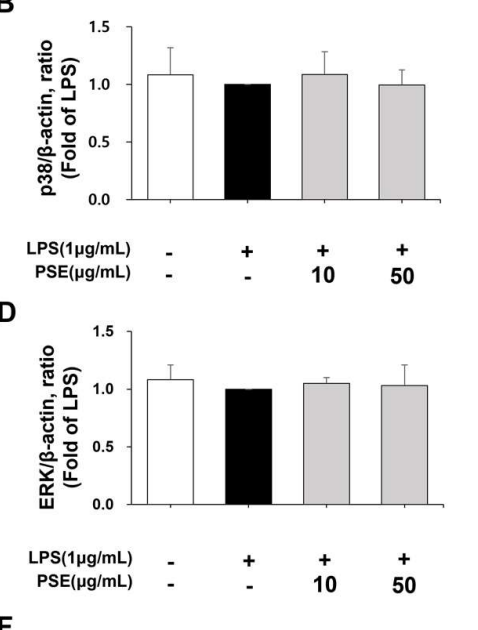

$\mathbf{F}$

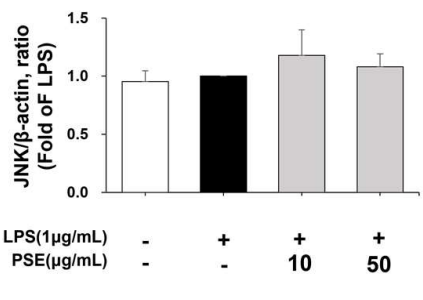

H
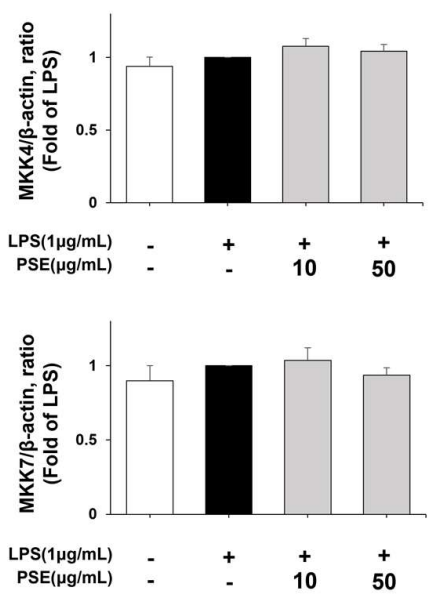

C

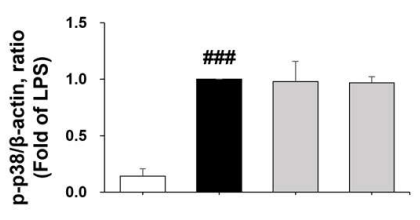

$\begin{array}{ccccc}\mathrm{LPS}(1 \mu \mathrm{g} / \mathrm{mL}) & - & + & + & + \\ \mathrm{PSE}(\mu \mathrm{g} / \mathrm{mL}) & - & - & 10 & 50\end{array}$

E

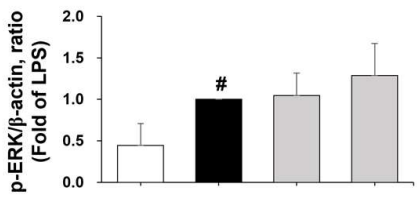

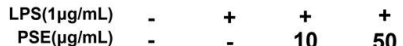

G

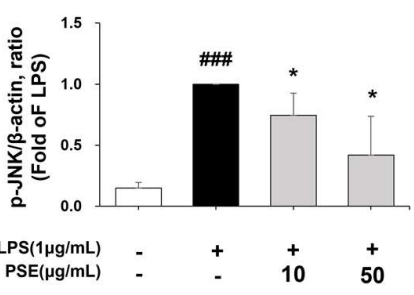

I

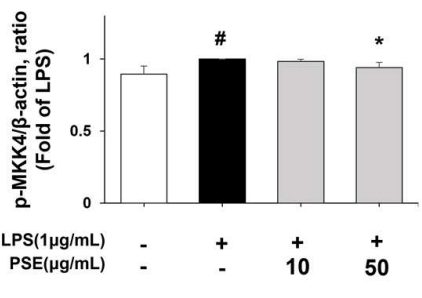

K

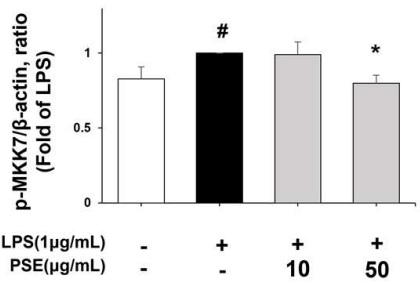


Figure 6. Inhibitory effect of PSE on the activation of MKK/MAPKs signaling. Expression of MKK/MAPKs signaling were determined by western blot analysis (A). The cells were pretreated $1 \mu \mathrm{g} / \mathrm{mL}$ LPS for $1 \mathrm{~h}$ and treated with 1-50 $\mu \mathrm{g} / \mathrm{mL}$ PSE for $3 \mathrm{~h}$ (B-G). The cells were pretreated 1-50 $\mu \mathrm{g} / \mathrm{mL}$ PSE for 30 minute and treated $1 \mu \mathrm{g} / \mathrm{mL}$ LPS for 60 minute $(\mathrm{H}-\mathrm{K})$. Data are presented as mean $\pm \mathrm{SD}$ from three independent experiments $(\# p<0.05$, \#\# $p<0.001$ versus the control group; ${ }^{*} p<0.05$ versus the LPS group).

\section{Discussion}

Inflammation occurs by fighting for the eradication of invading bacteria. Toll-like receptor 4 (TLR4) belongs to the TLR family of receptors that induce a pro-inflammation response to pathogens like LPS [25]. Activation of TLR4 and CD14 receptors play a critical role in the induction of inflammatory processes. Stimulating TLR4 induces activating various adaptor proteins, such as TIRAP and MyD88, and consecutively induces activation of NF- $\mathrm{B}$ and AP-1, leading to the transcription of inflammatory genes [26]. Excessive inflammation exacerbates pathological symptoms in various diseases including asthma, diabetes mellitus, inflammatory bowel disease, obesity, and sepsis [27]. Therefore, it is important to regulate inflammation for understanding inflammatory diseases and developing medications, and one of potential strategies for regulation of inflammation is to inhibit the signaling pathways of transcription factors such as NF-kB and AP-1 [28].

Lichen is a symbiotic cyanobiont, photobiont, and mycobiont [29]. Lichen extracts have the ability for antioxidant, antiviral, antitumor, and anti-inflammatory activities under experimental conditions [30]. Endolichenic fungi are ubiquitous in the environment and have been investigated for their antimicrobial, anti-neuroinflammatory, and anti-cancer effects [31-34].

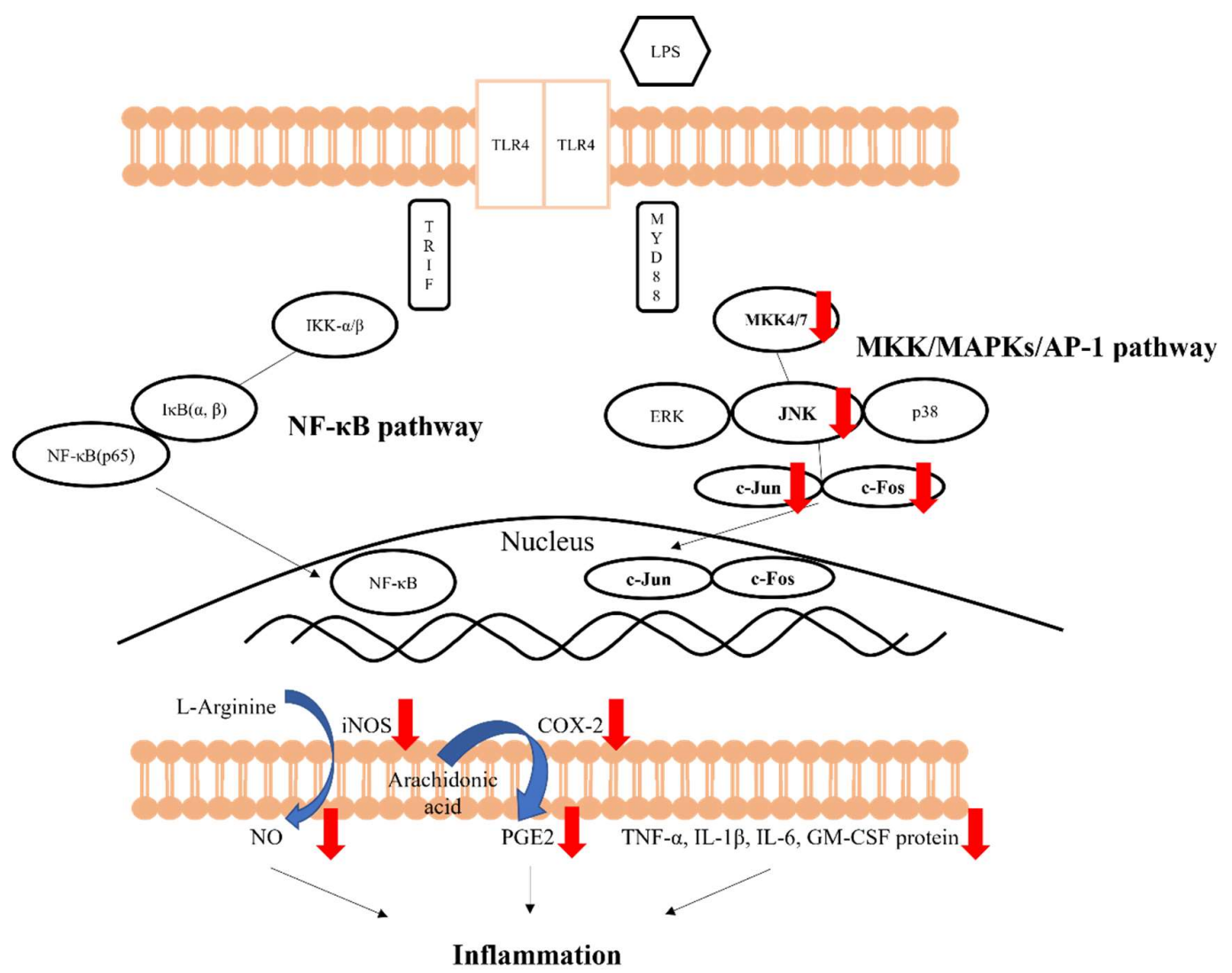

Figure 7. Schematic representation of the anti-inflammatory effects of phlebia sp. extract in RAW 264.7 macrophages. 
This study aimed to investigate the anti-inflammatory effects of Phlebia sp. extract (PSE). Inflammation, which is affected by expression levels of iNOS and COX-2, produces large amounts of the inflammatory effector molecules, NO, PEG2, and cytokines. [35]. Increased inflammatory cytokines make synergic effects to exacerbate inflammation. TNF$\alpha$ is critical for the synergistic induction of $\mathrm{NO}$ synthesis in macrophages stimulated by IFN- $\gamma$ or LPS. In addition, TNF- $\alpha$ induces several physiological effects, such as inflammation, cytotoxicity, septic shock, and cachexia, and IL-6 is believed to be an endogenous mediator of LPS-induced fever [6,35]. GM-CSF also upregulates the expression of TLR2, TLR4, and CD14 [13].

We investigated whether PSE attenuates inflammatory reaction in the LPS-stimulated macrophages by inhibiting signaling pathway MAPKs, AP-1, and NF- $\kappa$ B. In this study, we confirmed that PSE significantly inhibited the production of inflammatory cytokines including TNF- $\alpha$, IL-1 $\beta$, IL-6, and GM-CSF. MAPKs signaling is necessary to secrete cytokines during inflammation. We observed the activated-macrophages by LPS induces increased phosphorylation of MAPKs family proteins whereas PSE inhibits MAPKs, particularly JNK, and upstream pathway, MMK4/7. Reduced MAPKs signaling leads downregulation of the AP-1 pathway by the decrease of phosphorylated c-Jun and c-Fos. Resultingly, inflammatory effector molecules NO and PEG2 correlate with expression patterns of the signaling pathways in company with reduced inflammatory cytokine levels.

\section{Conclusions}

PSE inhibits the production of NO, PGE2, and Pro-inflammation cytokines, such as TNF- $\alpha$, IL-6, IL-1 $\beta$, GM-CSF in LPS-stimulated macrophages by down-regulating expression of iNOS and COX-2. Those phenomena are affected by inhibitory effects of PSE at the phosphorylation of MKK4/7, JNK, and AP-1 pathways. Collectively, these results demonstrate that PSE has therapeutic potential and can be developed into a novel anti-inflammatory agent.

Supplementary Materials: The following are available o

Author Contributions: E.H.L. and S.-T.Y. conceived and designed the experiments; E.H.L. and S.K.M. performed the experiments and analyzed the data; J.-J.K. and D.-J.C. contributed reagents/materials/analysis tools and wrote the paper. All authors have read and agreed to the published version of the manuscript.

Funding: This research received no external funding.

Institutional Review Board Statement: Not applicable.

Informed Consent Statement: Not applicable

Acknowledgments: This study was carried out with the support of The Korean National Research Resource Center Program (NRF-2017M3A9B8069471).

Conflicts of Interest: The authors declare no conflict of interest.

\section{References}

1. Surayot, U.; Wangtueai, S.; You, S.; Palanisamy, S.; Krusong, W.; Brennan, C.S.; Barba, F.J.; Phimolsiripol, Y.; Seesuriyachan, P. Extraction, Structural Characterisation, and Immunomodulatory Properties of Edible Amanita Hemibapha Subspecies Javanica (Corner and Bas) Mucilage Polysaccharide as a Potential of Functional Food. J. Fungi 2021, 7, 683.

2. Doherty, T.M. T-Cell Regulation of Macrophage Function. Curr. Opin. Immunol. 1995, 7, 400-404.

3. Yuan, R.; Huang, L.; Du, L.-J.; Feng, J.-F.; Li, J.; Luo, Y.-Y.; Xu, Q.-M.; Yang, S.-L.; Gao, H.; Feng, Y.-L. Dihydrotanshinone Exhibits an Anti-Inflammatory Effect in Vitro and in Vivo through Blocking TLR4 Dimerization. Pharmacol. Res. 2019, 142, 102-114.

4. Noh, J.-I.; Mun, S.-K.; Lim, E.H.; Kim, H.; Chang, D.-J.; Hur, J.-S.; Yee, S.-T. Induction of Apoptosis in MDA-MB-231 Cells Treated with the Methanol Extract of Lichen Physconia Hokkaidensis. J. Fungi Basel Switz. 2021, 7, 188.

5. Castello, M.; Skert, N. Evaluation of Lichen Diversity as an Indicator of Environmental Quality in the North Adriatic Submediterranean Region. Sci. Total Environ. 2005, 336, 201-214. 
6. $\quad$ Mun, S.-K.; Kang, K.-Y.; Jang, H.-Y.; Hwang, Y.-H.; Hong, S.-G.; Kim, S.-J.; Cho, H.-W.; Chang, D.-J.; Hur, J.-S.; Yee, S.-T. Atraric Acid Exhibits Anti-Inflammatory Effect in Lipopolysaccharide-Stimulated RAW264.7 Cells and Mouse Models. Int. J. Mol. Sci. 2020, 21, E7070.

7. Molnár, K.; Farkas, E. Current Results on Biological Activities of Lichen Secondary Metabolites: A Review. Z. Naturforschung C J. Biosci. 2010, 65, 157-173.

8. Hwang, Y.-H.; Lee, S.-J.; Kang, K.-Y.; Hur, J.-S.; Yee, S.-T. Immunosuppressive Effects of Bryoria Sp. (Lichen-Forming Fungus) Extracts via Inhibition of CD8+ T-Cell Proliferation and IL-2 Production in CD4+ T Cells. J. Microbiol. Biotechnol. 2017, 27, 11891197.

9. Shen, S.; Ma, X.; Xu, T.-M.; ZHAO, C.-L. Phlebia Ailaoshanensis Sp. Nov. (Polyporales, Basidiomycota) Evidenced by Morphological Characters and Phylogenetic Analyses. Phytotaxa 2018, 373, 184.

10. Wang, Z.; Mu, W.; Li, P.; Liu, G.; Yang, J. Anti-Inflammatory Activity of Ortho-Trifluoromethoxy-Substituted 4-PiperidioneContaining Mono-Carbonyl Curcumin Derivatives in Vitro and in Vivo. Eur. J. Pharm. Sci. 2021, 160, 105756.

11. Le, H.T.T.; Cho, Y.-C.; Cho, S. Methanol Extract of Guettarda Speciosa Linn. Inhibits the Production of Inflammatory Mediators through the Inactivation of Syk and JNK in Macrophages. Int. J. Mol. Med. 2018, 41, 1783-1791.

12. Xu, J.; Xiao, C.; Xu, H.; Yang, S.; Chen, Z.; Wang, H.; Zheng, B.; Mao, B.; Wu, X. Anti-Inflammatory Effects of Ganoderma Lucidum Sterols via Attenuation of the P38 MAPK and NF-KB Pathways in LPS-Induced RAW 264.7 Macrophages. Food Chem. Toxicol. 2021, 150, 112073.

13. Shiomi, A.; Usui, T. Pivotal Roles of GM-CSF in Autoimmunity and Inflammation. Mediators Inflamm. 2015, 2015 , e568543.

14. Kim, J.-Y.; Park, S.J.; Yun, K.-J.; Cho, Y.-W.; Park, H.-J.; Lee, K.-T. Isoliquiritigenin Isolated from the Roots of Glycyrrhiza Uralensis Inhibits LPS-Induced INOS and COX-2 Expression via the Attenuation of NF-KB in RAW 264.7 Macrophages. Eur. J. Pharmacol. 2008, 584, 175-184.

15. Chen, X.; Zong, C.; Gao, Y.; Cai, R.; Fang, L.; Lu, J.; Liu, F.; Qi, Y. Curcumol Exhibits Anti-Inflammatory Properties by Interfering with the JNK-Mediated AP-1 Pathway in Lipopolysaccharide-Activated RAW264.7 Cells. Eur. J. Pharmacol. 2014, 723, 339-345.

16. Jeon, K.-I.; Xu, X.; Aizawa, T.; Lim, J.H.; Jono, H.; Kwon, D.-S.; Abe, J.; Berk, B.C.; Li, J.-D.; Yan, C. Vinpocetine Inhibits NF-KBDependent Inflammation via an IKK-Dependent but PDE-Independent Mechanism. Proc. Natl. Acad. Sci. 2010, 107, 9795-9800.

17. Lee, H.-S. Orostachys Japonicus Extract Inhibits the Lipopolysaccharide-Induced pro-Inflammatory Factors by Suppression of Transcription Factors. Food Sci. Nutr. 2020, 8, 1812-1817.

18. Oh, C.-H.; Park, S.-Y.; Han, J.-S. Phospholipase D1 Is Required for Lipopolysaccharide-Induced Tumor Necrosis Factor- $\alpha$ Expression and Production through S6K1/JNK/c-Jun Pathway in Raw 264.7 Cells. Cytokine 2014, 66, 69-77.

19. Hop, H.T.; Arayan, L.T.; Huy, T.X.N.; Reyes, A.W.B.; Vu, S.H.; Min, W.; Lee, H.J.; Rhee, M.H.; Chang, H.H.; Kim, S. The Key Role of C-Fos for Immune Regulation and Bacterial Dissemination in Brucella Infected Macrophage. Front. Cell. Infect. Microbiol. 2018, 8, 287.

20. Lee, W.-S.; Shin, J.-S.; Jang, D.S.; Lee, K.-T. Cnidilide, an Alkylphthalide Isolated from the Roots of Cnidium Officinale, Suppresses LPS-Induced NO, PGE2, IL-1 $\beta$, IL-6 and TNF- $\alpha$ Production by AP-1 and NF-KB Inactivation in RAW 264.7 Macrophages. Int. Immunopharmacol. 2016, 40, 146-155.

21. Li, S.; Dai, Q.; Zhang, S.; Liu, Y.; Yu, Q.; Tan, F.; Lu, S.; Wang, Q.; Chen, J.; Huang, H.; et al. Ulinastatin Attenuates LPS-Induced Inflammation in Mouse Macrophage RAW264.7 Cells by Inhibiting the JNK/NF-KB Signaling Pathway and Activating the PI3K/Akt/Nrf2 Pathway. Acta Pharmacol. Sin. 2018, 39, 1294-1304.

22. Kang, Y.S.; Chung, Y.C.; Lee, J.N.; Kim, B.S.; Hyun, C.-G. Anti-Inflammatory Effects of 6,7-Dihydroxy-4-Methylcoumarin on LPS-Stimulated Macrophage Phosphorylation in MAPK Signaling Pathways. Nat. Prod. Commun. 2021, 16, $1934578 X 211020970$.

23. Wang, X.; Destrument, A.; Tournier, C. Physiological Roles of MKK4 and MKK7: Insights from Animal Models. Biochim. Biophys. Acta BBA - Mol. Cell Res. 2007, 1773, 1349-1357.

24. Lee, N.; Heo, Y.J.; Choi, S.-E.; Jeon, J.Y.; Han, S.J.; Kim, D.J.; Kang, Y.; Lee, K.W.; Kim, H.J. Anti-Inflammatory Effects of Empagliflozin and Gemigliptin on LPS-Stimulated Macrophage via the IKK/NF-KB, MKK7/JNK, and JAK2/STAT1 Signalling Pathways. J. Immunol. Res. 2021, 2021, e9944880.

25. Ciesielska, A.; Matyjek, M.; Kwiatkowska, K. TLR4 and CD14 Trafficking and Its Influence on LPS-Induced pro-Inflammatory Signaling. Cell. Mol. Life Sci. 2021, 78, 1233-1261.

26. Badshah, H.; Ali, T.; Kim, M.O. Osmotin Attenuates LPS-Induced Neuroinflammation and Memory Impairments via the TLR4/NFкB Signaling Pathway. Sci. Rep. 2016, 6, 24493.

27. Wang, G.; Wang, J.; Xin, C.; Xiao, J.; Liang, J.; Wu, X. Inflammatory Response in Epilepsy Is Mediated by Glial Cell Gap Junction Pathway (Review). Mol. Med. Rep. 2021, 24, 1-5.

28. Wei, H.; Shi, Y.; Yuan, Z.; Huang, Z.; Cai, F.; Zhu, J.; Zhang, W.; Li, J.; Xiong, Q.; Wang, Y.; et al. Isolation, Identification, and Anti-Inflammatory Activity of Polysaccharides of Typha Angustifolia. Biomacromolecules 2021, 22, 2451-2459.

29. Nguyen, H.; Ketha, A.; Kukavica, B.; Tatipamula, V. Anti-Inflammatory Potential of Lichens and Its Substances. In; 2021; pp. 19.

30. Tripathi, A.H.; Negi, N.; Gahtori, R.; Kumari, A.; Joshi, P.; Tewari, L.M.; Joshi, Y.; Bajpai, R.; Upreti, D.K.; Upadhyay, S.K. A Review of Anti-Cancer and Related Properties of Lichen-Extracts and Metabolites. Anticancer Agents Med. Chem. 2021.

31. Ting, A.S.Y.; Cheng, C.K.W.; Santiago, K.A.A. Decolourization of Malachite Green Dye by Endolichenic Fungi from the Lichen Usnea Sp.: A Novel Study on Their Dye Removal Potential. J. King Saud Univ. - Sci. 2021, 33, 101579. 
32. Padhi, S.; Masi, M.; Cimmino, A.; Tuzi, A.; Jena, S.; Tayung, K.; Evidente, A. Funiculosone, a Substituted Dihydroxanthene-1,9Dione with Two of Its Analogues Produced by an Endolichenic Fungus Talaromyces Funiculosus and Their Antimicrobial Activity. Phytochemistry 2019, 157, 175-183.

33. Zhai, Y.-J.; Li, J.-N.; Gao, Y.-Q.; Gao, L.-L.; Wang, D.-C.; Han, W.-B.; Gao, J.-M. Structurally Diverse Sesquiterpenoids with AntiNeuroinflammatory Activity from the Endolichenic Fungus Cryptomarasmius Aucubae. Nat. Prod. Bioprospecting 2021, 11, 325332.

34. Yang, Y.; Bae, W.K.; Nam, S.-J.; Jeong, M.-H.; Zhou, R.; Park, S.-Y.; Taş, İ.; Hwang, Y.-H.; Park, M.-S.; Chung, I.J.; et al. Acetonic Extracts of the Endolichenic Fungus EL002332 Isolated from Endocarpon Pusillum Exhibits Anticancer Activity in Human Gastric Cancer Cells. Phytomedicine 2018, 40, 106-115.

35. Kim, J.-B.; Han, A.-R.; Park, E.-Y.; Kim, J.-Y.; Cho, W.; Lee, J.; Seo, E.-K.; Lee, K.-T. Inhibition of LPS-Induced INOS, COX-2 and Cytokines Expression by Poncirin through the NF-KB Inactivation in RAW 264.7 Macrophage Cells. Biol. Pharm. Bull. 2007, 30, 2345-2351. 